\title{
Reirradiation in Head and Neck Cancer: A Curative Intent in Recurrence or Second Tumors
}

Enrique Jurado Martín ${ }^{1 *}$, Vladimir Suarezb ${ }^{2}$, Ismael Herruzo Cabrera ${ }^{3}$, Lourdes de la Peña ${ }^{3}$, Gustavo Ossola Lentatia ${ }^{1}$, Maria José Ortiz Gordillob ${ }^{2}$ and Jerónimo Pachon Ibañezb²

${ }^{1}$ Radiation Oncology Unit, RiojaSalud Foundation-San Pedro Hospital, La Rioja, Spain

${ }^{2}$ Radiation Oncology Department, Virgen del Rocio University Hospital, Seville, Spain

${ }^{3}$ Department of Radiology and Physical Medicine, Ophthalmology and Otorhinolaryngology Málaga University, Málaga, Spain

*Corresponding author: Enrique Jurado Martín, Radiation Oncology Unit, Rioja Salud Foundation -San Pedro Hospital, La Rioja, Spain, Tel:+63 82 $2214950 ;$ E-mail: ogata@k-mcc.net

Received date: January 29, 2016, Accepted date: February 16, 2016, Publication date: February 23, 2016

Copyright: ( 2015 Martín EJ, et al. This is an open-access article distributed under the terms of the Creative Commons Attribution License; which permits unrestricted use; distribution; and reproduction in any medium; provided the original author and source are credited.

\begin{abstract}
Purpose: To assess the efficacy and safety of reirradiation in head and neck cancer and potential prognostic factors associated.

Material and methods: Cohort study of patients treated with curative reirradiation for recurrence or second primary tumor. The analysis of RR is obtained prospectively from the database available at both centers. Statistical analysis was performed using the R Commander 2.0 software.

Results: Between 2006 and 2013, 40 patients with head and neck carcinoma were reirradiated. The mean dose was $66.39 \mathrm{~Gy} .35 \%$ of patients showed acute toxicity grade 3 or higher and $20.5 \%$ showed chronic toxicity grade 2 or higher. The median follow-up was 11 months. The overall survival at 2 years was $41 \%$; disease free survival and locoregional control at 1 year was $35.9 \%$ and $41.1 \%$ respectively. The time between treatments and disease-free interval to death and local recurrence were statistically significant $(p<0.05)$; and the first treatment scheme to distant metastases.
\end{abstract}

Keywords: Reirradiation; Head and neck; Cancer; Retreatment

\section{Introduction}

The reirradiation (RR) in head and neck cancer is one of the most controversial points raised in multidisciplinary committees. In the meta-analysis of Blandchard between $46.5 \%$ to $60 \%$ of patients will have locoregional recurrence. The cause of death is more closely related to the locoregional failure that to the distant metastasis, $50-60 \%$ of these patients die due to persistent or recurrent loco-regional disease and $70-90 \%$ of those who develop distant metastases also have loco-regional disease. In addition, survivors have a high incidence of second primary tumors with a rate of $14.2 \%$. In these cases, treatment options are limited. The treatment of choice in relapse or second neoplasms in previously irradiated areas is salvage surgery, with survival rates at 5 years of up to $39 \%$. In inoperable tumors and/or unresectable cases, chemotherapy (CT) can be considered as an alternative, although with discrete results, with response rates of approximately $30 \%$ and a median survival of 5 to 6 months. It should be noted that the results obtained with cisplatin, 5-FU plus cetuximab, achieve response rates of $36 \%$, an overall survival of 10.1 months and progression-free survival of 5.6 months. The RR in head and neck tumors is not the treatment of choice [1], however, it has gained acceptance for patients with recurrent or unresectable seconds tumors and in cases with poor prognostic factors. In this prospective study, we evaluate the efficacy and safety of reirradiation in head and neck cancer and potential prognostic factors associated with it.

\section{Material and Methods}

This is a two-way cohorts study in which patients were treated with curative intent by $\mathrm{RR}$ of head and neck carcinoma because of recurrence or second primary tumor. Patients were treated in the Radiation Oncology Department, Virgen del Rocio University Hospital (HUVR), Seville and Radiation Oncology Unit, Rioja Salud Foundation-San Pedro Hospital (FRS-HSP), La Rioja. RR data were obtained prospectively from both centers. First irradiation data was collected retrospectively in some cases. The inclusion and exclusion criteria, as well as the different variables analyzed are shown in Table 1.

\begin{tabular}{|l|l|}
\hline Inclusion criteria & Exclusion criteria \\
\hline $\begin{array}{l}\text { Patients with recurrent head and neck cancer or second tumor in the head and neck area, } \\
\text { previously irradiated. }\end{array}$ & Patients with poor performance status \\
\hline Patient with good performance status, ECOG 0-1 & Existence prior chronic toxicity important (>G3) \\
\hline
\end{tabular}


Citation: Martín EJ, Suarezb V, Cabrera IH, Peña LDL, Lentatia GO, et al. (2016) Reirradiation in Head and Neck Cancer: A Curative Intent in Recurrence or Second Tumors. J Nucl Med Radiat Ther 7: 281. doi:10.4172/2155-9619.1000281

Page 2 of 7

\begin{tabular}{|l|l|}
\hline Time since first irradiation at least 6 months. & Cumulative doses greater than 54 Gy spinal \\
\hline & Existence of distant metastases \\
\hline Analyzed Variables & Disease-free interval \\
\hline Location of the primary tumor & Variation between initial histological grade and tumor recurrence or \\
\hline Staging of the first disease & second primary \\
\hline First therapeutic scheme & Second therapeutic scheme \\
\hline Existence of recurrent disease that required other previous treatments (surgery or CT) & Indication of surgery. Prior to RR \\
\hline Second disease: local recurrence, nodal recurrence or second primary tumor & Display QT in the second treatment \\
\hline Interval between radiation treatments, IBT & Get lower or higher doses $60 \mathrm{~Gy}$ or 66 Gy \\
\hline
\end{tabular}

Table 1: Criteria for inclusion and exclusion. Analyzed variables.

Radiation therapy administered in the second course of radiotherapy (RT) was performed using three-dimensional conformal RT (3D) with high-energy photons. Dose per fraction was either 1.8 or 2 Gy. We considered the irradiation of the lower possible volume, to avoiding the radiation of elective nodal chains to minimize side effects and cumulated dose in risk organs.

Toxicities have been defined by CTCAE 3.0 (common terminology criteria for adverse events).

The statistical analysis was performed using the R Commander 2.0 software. Kaplan-Meier method was used for the univariate survival analysis and Cox regression was used for the multivariate survival analysis.

\section{Results}

Between September 2006 and September 2013, a total of 40 patients with head and neck carcinoma were re-irradiated; of which 33 were from HUVR and 7 from FRS-HSP. Data from the first and second treatment are summarized in Table 2.

\begin{tabular}{|c|c|c|}
\hline \multicolumn{3}{|c|}{ Number (\%) } \\
\hline \multirow[t]{2}{*}{ Sex } & Women & $7(17.5)$ \\
\hline & Men & $33(82.5)$ \\
\hline \multicolumn{2}{|c|}{ Mean/median age } & $59.2 / 62$ (DS 11.83) \\
\hline \multicolumn{3}{|c|}{ Localization } \\
\hline & Pharynx & $7(17.5)$ \\
\hline & Larynx & $18(45)$ \\
\hline & Oral Cavity & $9(22.5)$ \\
\hline & Unknown primary & $4(10)$ \\
\hline & Others & $2(5)$ \\
\hline \multirow[t]{3}{*}{ Histology } & Epidermoid & $36(90)$ \\
\hline & undifferentiated & $2(5)$ \\
\hline & Adenocarcinoma & $1(2.5)$ \\
\hline
\end{tabular}

\begin{tabular}{|l|l|l|}
\hline & Others & $1(2.5)$ \\
\hline T & Tx & $5(12.5)$ \\
\hline & T1-2 & $10(30.3)$ \\
\hline N & T3-4 & $18(54.54)$ \\
\hline & N0-1 & $19(54.28)$ \\
\hline & N2 & $13(37.14)$ \\
\hline Initial Stage & N3 & $3(8.57)$ \\
\hline & I & $4(11.76)$ \\
\hline & II & $2(5.88)$ \\
\hline Radiotherapy & III & $10(29.41)$ \\
\hline Toxicity & IV & $18(52.94)$ \\
\hline & Acute (>G3) & 65.94 Gy \\
\hline Treatment & Chronicle (>G2) & $9(22.5)$ \\
\hline Scheme & RT alone & $2(5)$ \\
\hline & & $5(12.5)$ \\
\hline Disease Free Interval & Surgery and RT & $13(32.5)$ \\
\hline & RTQT & $13(32.5)$ \\
\hline & Surgery and RTQT & $9(22.5)$ \\
\hline & & Mean 33.5 /Median 16 \\
\hline & & Mean 44.77/Median 23.5 \\
\hline
\end{tabular}

Table 2: Characteristics of patients during the first and second radiotherapy. Features of second treatment.

\section{Features of the first treatment}

The average age of the patients was 59.2 years (median 62 years). Most frequent location was the larynx (45\%) the histology squamous 
cell carcinoma in $90 \%$ of the patients. $82.35 \%$ of patients had advanced disease stages (III-IV).

$55 \%$ had undergone surgery prior to radiotherapy and $60 \%$ of patients had undergone CT. Most regimens used CT schemes based on platinum (23 patients) and in 1 patient cetuximab. The therapeutic regimens used in the first treatment was RT exclusively in 5 patients, RT and CT in 13 patients, surgery followed by RT in 13 patients and surgery, RT and CT in 9 patients. Radiotherapy was administered between June 1993 and October 2011. The radiotherapy technique used varied depending on the technology available at the time. 2D technique was performed in a total of 4 patients in HUVR until 2002. The rest of the patients were treated with three dimensional technique (3D).

The average dose administered was 65.94 Gy (standard deviation, SD 66.49) on the primary tumor and affected lymph nodes or surgical bed; and 50 Gy (SD 3.464) on elective lymph node chains. The fractionation employed was 2 Gy (5 times a week, conventional treatment), except in 4 patients treated with fractionation $1.8 \mathrm{~Gy}$. Acute toxicity grade 3 or higher showed in $52.5 \%$ of patients. Chronic toxicities G2 or higher after the first treatment were xerostomia and fibrosis, showed in $5 \%$ of patients.

\section{Diseases between treatments}

After this first RT, $27.5 \%$ had disease that was treated with surgery (6 patients), CT (2 patients) and both ( 3 patients), without RT. This first relapse occurred within was average of 31.09 months and a median of 14 months since the end of the first treatment (SD 33.55).

\section{Features of the second treatment}

Second radiation causes were: second primary tumor (27.5\%: stage IV 5, stage III 5 and stage II 1 patients), local recurrence or relapse $(47.5 \%)$ and nodal recurrence (25\%). The disease-free interval averaged 33.5 months (median 16 months, DS 46.69) and the mean interval between treatments (IBT) was 44.77 months (median 23.5 months, SD 53.26).

Treatment schemes were: RT alone in 11 patients, RT and CT in 14 patients, surgery followed by RT in 7 patients and surgery followed by $\mathrm{CT}$ and $\mathrm{RT}$ in 8 patients.

In this way $37.5 \%$ (15 patients) of patients were previously operated (microscopic and macroscopic residual disease, 4 and 4 patients) and $72.5 \%$ received CT. The CT schemes most commonly used were based on platinum (24 patients) and cetuximab (10 patients).

In terms of the administered radiotherapy, the average dose administered was $66.39 \mathrm{~Gy} /(\mathrm{SD} 6.24)$ on tumor lesion or surgical bed. In 9 patients the dose was less than or equal to $60 \mathrm{~Gy}$. The fractionation employed was $2 \mathrm{~Gy}$ (5 times a week, conventional treatment).

$35 \%$ of patients presented acute toxicity grade 3 or higher. Chronic toxicity G2 or higher was shown by $20.5 \%$ of patients: 5 fibrosis, 4 xerostomia and necrosis, hypothyroidism, trismus or odynophagia 1 respectively.

The median follow-up of patients was 11 months (SD 16.33), being 22 months in survivors (SD 22.13). At the end of the study, 16 patients were alive (11 without disease, 3 with local disease and 2 with metastases); 24 patients had died (14 with local disease, 7 local and distant disease, 1 with distant disease and 2 patients died of non-cancer causes without oncological disease). This relation between the deaths and the existence of local and/or distance disease was statistically significant $(\mathrm{p}<0.001)$.

\section{Analytical statistics}

Statistically significant variables $(\mathrm{p}<0.05)$ for local recurrence and death were the time between treatments and the disease-free interval. The average difference between both groups is indicated in Table 3 . The first treatment scheme was associated with distant metastases $(\mathrm{p}=$ 0.01 ). The rest of variables were not statistically significant. Univariate survival analysis (Figure 1).

\begin{tabular}{|c|c|c|c|c|c|c|}
\hline & \multicolumn{2}{|c|}{$\begin{array}{l}\text { Local } \\
\text { recurrence }\end{array}$} & \multicolumn{2}{|c|}{ Exitus } & \multicolumn{2}{|c|}{$\begin{array}{l}\text { Metastasi } \\
\text { s }\end{array}$} \\
\hline & No & Yes & Alive & $\begin{array}{l}\text { Death } \\
\text { s }\end{array}$ & No & Yes \\
\hline Disease Free Interval & 44 & 24.9 & 48.37 & 23.58 & 39.4 & 15.7 \\
\hline Interval between treatments & 60.16 & 32.18 & 69.81 & 28.08 & 53.4 & 18.9 \\
\hline
\end{tabular}

Table 3: Average of months in the variables that were statistically significant.

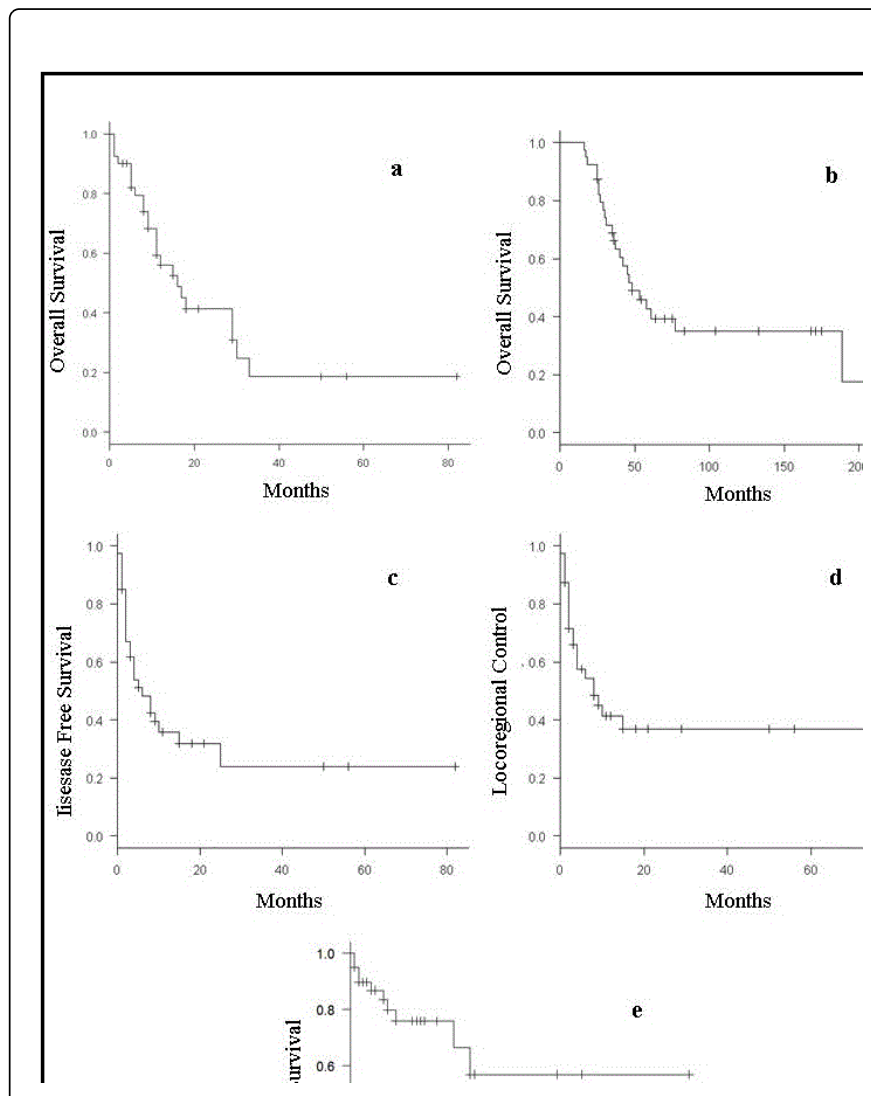

Figure 1: Kaplan Meier Survival. (a) Overall survival (OS) from the end of the second radiotherapy. (b) Overall survival (OS) from the end of the first radiotherapy. (c) Disease-free survival, DFS. (d) Loco-regional control, LRC. (e) Metastases-free survival, MFS.

Overall survival (OS): OS after RR has submitted a median of 16 months (95\% CI 11-29). The survival rate at 12 months was 56\% (95\% 
Citation: Martín EJ, Suarezb V, Cabrera IH, Peña LDL, Lentatia GO, et al. (2016) Reirradiation in Head and Neck Cancer: A Curative Intent in

CI $38-70.5), 41 \%$ at 24 months (95\% CI 24-57) and $18.6 \%$ at 36 months (95\% CI 5-37).

Disease-free survival (DFS): DFS has submitted a median of 6 months (95\% CI 2-15). The DFS at 6 months was $48.2 \%$ (95\% CI $31.8-62.9$ ), $35.9 \%$ at 12 months (95\% CI $20.7-51.4)$ and $31.9 \%$ at 15 months (95\% CI 17.1-47.8).

Loco-regional control (LRC): LCR is determined by the appearance of tumor disease in the irradiated area after RR. In our study, the median CLR at 6 months was $54.4 \%$ (95\% CI 37-68), $41.4 \%$ at 12 moths $(95 \%$ CI $24-57)$ and it remains at $36.8 \%$ at 15 months $(95 \%$ CI 24-57).

Metastasis-free survival (MFS): More than $50 \%$ of patients had no distance disease even after more than 25 months (55.7\%). The SLM after RR at 6 months was $86.8 \%$ (95\% CI 71.1-94.3) $75.9 \%$ at 12 moths (95\% CI 56.8-87.4) and $56.9 \%$ at 30 months (95\% CI 28.8-77.4).

The univariate survival analysis did not find any statistically significant variable (OS, DFS, LRC or MFS); with the exception of time between treatments, the cut-off point to the 12 months is significant ( $p$ $<0.05$ ) for the LRC and for the DFS (Figure 2). This was not the case when the cut was established at 24 months.

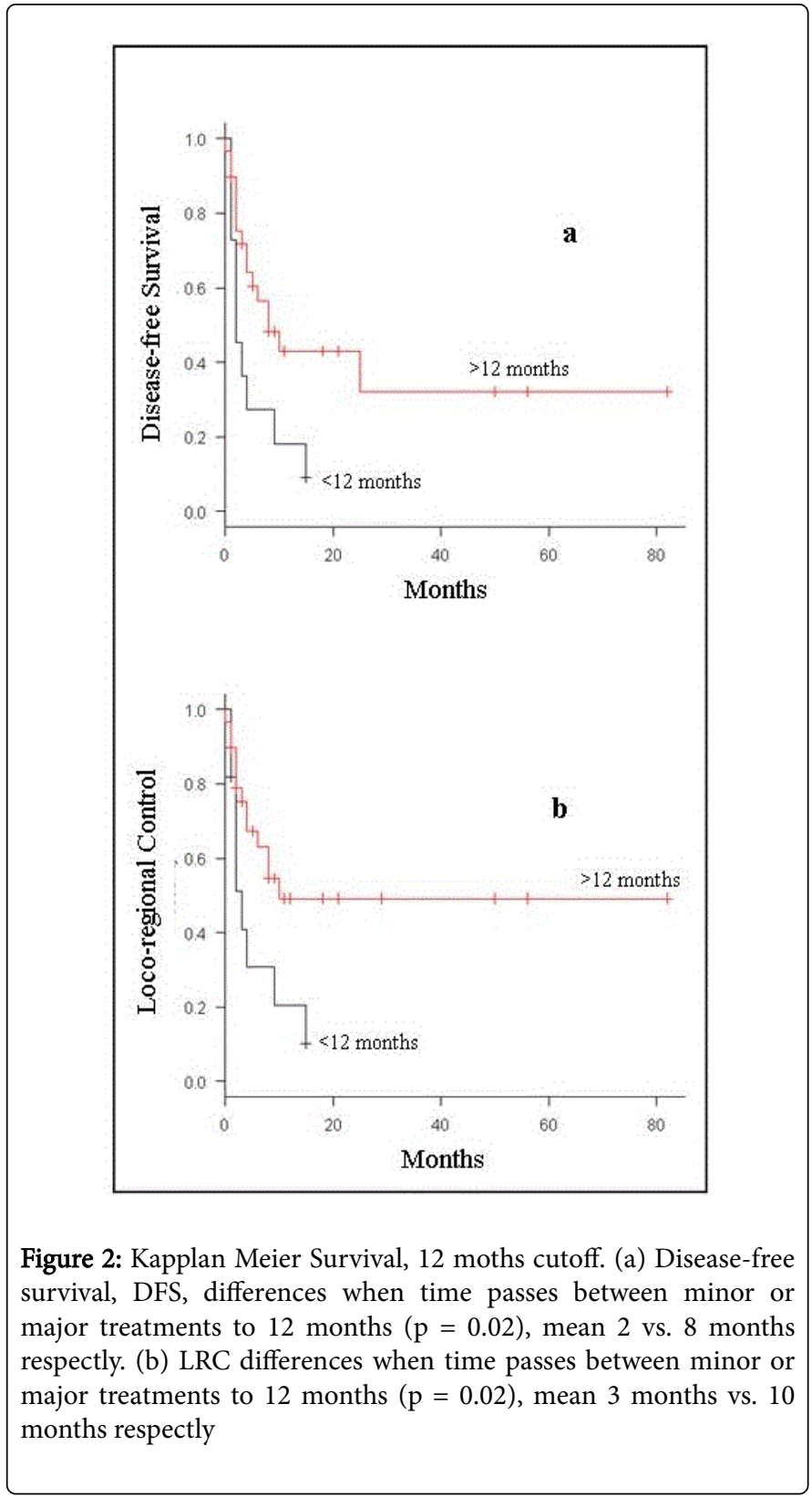

\section{Multivariate survival analysis}

Due to limited number of statistically significant variables in the univariate analysis, the multivariate analysis was performed for variables that had $\mathrm{p}<0.1$ and $\mathrm{p}<0.2$ (Table 4 ).

\begin{tabular}{|l|l|l|l|l|}
\hline \multicolumn{3}{|l|}{} & p < 0.1 & \multicolumn{2}{l|}{ p $<0.2$} \\
\cline { 2 - 6 } & HR & p & HR & p \\
\hline Overall Survival & 2.53 & 0.06 & 2.2 & 0.13 \\
\hline Doses >60 Gy & & & 1.6 & 0.3 \\
\hline Previous surgery to 2nd radiation & & & 1.03 & 0.1 \\
\hline Disease Free interval & 1.02 & 0.15 & & \\
\hline
\end{tabular}




\begin{tabular}{|c|c|c|c|c|}
\hline Interval between treatments & 0.9 & 0.12 & 0.9 & 0.09 \\
\hline \multicolumn{5}{|l|}{ Disease Free Survival } \\
\hline First treatment scheme & 1.14 & 0.56 & 1.05 & 0.82 \\
\hline Doses >60 Gy & 2.9 & 0.03 & 2.91 & 0.03 \\
\hline Previous surgery to 2 nd radiation & & & 1.44 & 0.44 \\
\hline QT in the 2nd treatment & & & 0.94 & 0.92 \\
\hline Second disease & & & 0.84 & 0.72 \\
\hline Disease Free interval & 0.97 & 0.11 & 1.01 & 0.23 \\
\hline Interval between treatments & 1.01 & 0.35 & 0.97 & 0.09 \\
\hline \multicolumn{5}{|l|}{ Locoregional Control } \\
\hline Doses >60 Gy & 3.12 & 0.04 & 3.2 & 0.03 \\
\hline Previous surgery to 2 nd radiation & & & 2.1 & 0.1 \\
\hline QT in the 2nd treatment & & & 1.4 & 0.5 \\
\hline Disease Free interval & 1.01 & 0.41 & 1.01 & 0.25 \\
\hline Interval between treatments & 0.98 & 0.14 & 0.98 & 0.53 \\
\hline \multicolumn{5}{|l|}{ Metastases Free Survival } \\
\hline Second disease & & & 0.2 & 0.02 \\
\hline First Disease localization & & & 0.8 & 0.46 \\
\hline First treatment scheme & & & 3.5 & 0.007 \\
\hline
\end{tabular}

Table 4: Multivariate analysis, Cox regression, HR: Hazard Ratio.

Where we have used only variables $\mathrm{p}<0.1,>60$ Gy versus $<60$ Gy was the only statistically significant variable in terms of the MFS and LRC, with a hazard ratio (HR) of 2.9 and 3.12 respectively. If we use the variables with $\mathrm{p}<0.2$, in addition to $>60 \mathrm{~Gy}$, the statistically significant variables were second disease and therapeutic scheme received in the first disease in terms of MFS, with a HR of 0.2 and 3.5 respectively.

\section{Discussion}

In this study, we have analyzed 40 patients who have been irradiated a second time in the head and neck area. The treatment of choice should be the surgery in case of relapse and the impossibility of this option we will be considered the RR. In addition, the majority of patients who relapse or suffer from a second primary tumor in this area tend to have comorbidities, secondary sequelae of previous treatments (surgery, CT or RT) or of the current disease. All these situations lead to a low "performance status" that contraindicates the possibility of RR. Due to all these situations, the number of patients obtained for the study was low although similar to other studies [2]. The improved outcome by the chemotherapy [3] results a large number of patients who exceed the 6 months interval between irradiation which is the recommended minimum time, thus this could mean an increase in the number of re-irradiations.

The risk of causing irreparable damage is the biggest problem of this treatment and this may influence the quality of life and even survival rate of patients. The use of the most modern techniques of RT

(intensity modulated radiation therapy (IMRT) and radiosurgery) have demonstrated reliability and effectiveness in these circumstances and may improve the outcome and quality of life of these patients. In our environment, the technique of treatment available was threedimensional radiotherapy. In our cohort of patients largely heterogeneous, due to the initial staging, initial location, characteristics of the second disease (local recurrence vs. nodal recurrence vs. second primary), treatment regimens employees (RT, surgery + RT, RTCT or surgery + RTCT), etc., this we consider as a limitation for statistical analysis. Although, this heterogeneity disappears with to the characteristics of radiotherapy employed.

A confounding factor in assessing the results is the treatment carried out between treatments, in what we call the intermediate disease $(27.5 \%$ of patients). These patients were treated at that time, either with surgery or with CT or both; and its influence was difficult to assess on the second radiation. We can conclude that the means employed were insufficient.

In our study, few patients received elective nodal RT (4 patients) in the second treatment, by protocol these patients only if they had not been previously dealt with it. The rate of severe toxicities $>$ grade 3 in the second treatment, acute (35\%) or chronic (20.5\%), have not been higher than the reflected in the literature despite the fact that the treatment was performed using three dimensional radiation therapy.

Several studies have suggested prognostic factor, such as tumor size, resectability, the IBT radiotherapy, whether it is second or relapse tumor, anatomic localization or the administered dose as statistically significant factors. In the inferential analysis of our study, the only factors found to be statistically significant were the time between treatments and disease-free interval. These factors have been endorsed by the literature as prognostic factors [4-9]. It is shown that the greater time between treatments the greater DFS and the better LRC, but a greater time doesn't mean a better DFS. There is another factor that was statistically significant in this analysis: the first therapeutic scheme and its relation with the developing distant metastasis $(\mathrm{p}=0.01)$. This situation has not been analyzed in the literature reviewed, so we believe it is the first time that it has been demonstrated. In our analysis, the group treated with surgery, CT and RT was the less favorable. We believe that the explanation is that this group had worse prognostic factors (positive margins, high histologic grade, and capsule overflow). We can consider this a significant variable, due to the possible existence of micrometastases, not clinically demonstrable, prior to the second course of RT. This assertion, however, must be considered with caution and does not mean this group of patients is not candidate for RR. More studies are needed to confirm this result.

22 of the 24 deceased patients had disease at that time, 21 with local disease, 7 of them also with distant disease and only 1 with distant disease exclusively; therefore this deaths you can be related to the progression of their disease $(\mathrm{p}<0.001)$. This leads us to think that what compromises the disease-free survival is the locoregional control. Therefore, we believe that improving treatments of local disease we will have higher rates of disease-free survival that would involve higher overall survival rates.

The study shows acceptable rates of survival and loco-regional control, despite the fact that the technique used was the threedimensional RT. The OS at two years has been $41 \%$ and the LRC at 1 year was $41.4 \%$. These rates are within the ranges of survival rate found in the literature [4-11]. The MFS (75.9\% at1 year) obtained high rates; this figure is not evaluable because patients die of local complications 
before they could develop distant metastases, which reaffirms the importance of local treatment for these patients.

In the survival analysis by subgroups, no significant differences were found, although there are other better prognostic factors endorsed by the literature within the disease is a second primary tumor (Steven and Spencer[12]), there was a possibility of surgery before radiotherapy (Salama) or when RR achieved high dose (Choe).

The only factor has been demonstrated to be statistically significant in relation to DFS and LRC has been the IBT, when stratified the cohort of patients between irradiation (less than or greater than 12 months) our test shown that the greater the time between treatments, the greater the survival rate (Table 3). For the MFS, this variable was not significant. The relation of time between treatments coincides with data published in other studies, where significant differences were observed in survival rate remain favorable for groups in which more time had passed between treatments. This result is supported by numerous studies [4-16]. Therefore, we recommend our series based on the range of 12-month interval such as a favorable prognostic factor to take into account when RR in head and neck carcinoma is indicated.

Multivariate analysis was performed in two circumstances, when the variables were obtained in the univariate $p<0.1$ and when they were obtained in the univariate $\mathrm{p}<0.2$, due to the low number of patients and the initially few significant variables. Therefore, we must acknowledge that in the multivariate analysis save confounding factors not adequately controlled may be found.

Under this premise, in our series, the only statistically significant variable was reaching higher dose than $60 \mathrm{~Gy}$ in the RR analysis to the LRC $\mathrm{p}<0.04$ and $\mathrm{p}<0.03$ for both analysis respectively and DFS $\mathrm{p}<$ 0.03 in the two analysis. Reaching high doses of radiation has been already been demonstrated by different studies [5-18] to be prognostic factor for the improvement of LRC and OS, so our results are consistent.

The second disease variable $(\mathrm{p}<0.02)$ and treatment scheme applied in the first disease variable $(p<0.007)$ were statistically significant a regard MFS, but only in the univariate analysis of these variables had a $\mathrm{p}<0.02$. In the analysis of the second disease, we have to be noted that the number of patients included in each subgroup is low. This more a clearly established prognostic factor in other studies [7-20]. Finally, the treatment scheme received in the first disease continues to demonstrate significant. This reaffirms the possibility of micrometastases prior to the second course of radiation. In the rest variables were not found to have clinical relevance which may be due to the small sample obtained for the study.

\section{Conclusions}

The RR in head and neck cancer shows good rates of OS, DFS and LRC with acceptable toxicities. Probably, improving the LRC will results in a improvement of OS. Of the variables studied, the interval between treatments and disease-free interval were the most important prognostic factors.

All these results must be interpreted with caution considering the statistical limitations described above, basically due to the size of the sample and the heterogeneity that this clinical situation presents. We believe there is a need for convenient multi-institutional studies which will increase the statistical power to establish more clearly the role of reirradiation in head and neck carcinoma [14,21-29].

\section{Conflicts of Interest}

The authors declare no conflict of interest.

Ethical approval: All procedures performed in studies involving human participants were in accordance with the ethical standards of the institutional and/or national research committee and with the 1964 Helsinki declaration and its later amendments or comparable ethical standards. For this type of study formal consent is not required

\section{References}

1. Blanchard P, Baujat B, Holostenco V, Bourredjem A, Baey C, et al. (2011) Meta-analysis of chemotherapy in head and neck cancer (MACH-NC): a comprehensive analysis by tumour site. Radiother Oncol 100: 33-40.

2. DeConti RC, Schoenfeld D (1981) A randomized prospective comparison of intermittent methotrexate, methotrexate with leucovorin, and a methotrexate combination in head and neck cancer. Cancer 48: 1061-1072.

3. Hong WK, Bromer RH, Amato DA, Shapshay S, Vincent M, et al. (1985) Patterns of relapse in locally advanced head and neck cancer patients who achieved complete remission after combined modality therapy. Cancer 56: 1242-1245.

4. Fontanesi J, Hetzler D, Ross J (1989) Effect of dose rate on local control and complications in the reirradiation of head and neck tumors with interstitial iridium-192. Int J Radiat Oncol Biol Phys 17: 365-369.

5. Kotwall C, Sako K, Razack MS, Rao U, Bakamjian V, et al. (1987) Metastatic patterns in squamous cell cancer of the head and neck. Am J Surg 154: 439-442.

6. Nishijima W, Takooda S, Tokita N, Takayama S, Sakura M (1993) Analyses of distant metastases in squamous cell carcinoma of the head and neck and lesions above the clavicle at autopsy. Arch Otolaryngol Head Neck Surg 119: 65-68.

7. Haughey BH, Gates GA, Arfken CL, Harvey J (1992) Meta-analysis of second malignant tumors in head and neck cancer: the case for an endoscopic screening protocol. Ann Otol Rhinol Laryngol 101: 105-112.

8. Goodwin WJ Jr (2000) Salvage surgery for patients with recurrent squamous cell carcinoma of the upper aerodigestive tract: when do the ends justify the means? Laryngoscope 110: 1-18.

9. Browman GP, Cronin L (1994) Standard chemotherapy in squamous cell head and neck cancer: what we have learned from randomized trials. Semin Oncol 21: 311-319.

10. Hong WK, Schaefer S, Issell B, Cummings C, Luedke D, et al. (1983) A prospective randomized trial of methotrexate versus cisplatin in the treatment of recurrent squamous cell carcinoma of the head and neck. Cancer 52: 206-210.

11. Gibson MK, Li Y, Murphy B, Hussain MH, DeConti RC, et al. (2005) Randomized phase III evaluation of cisplatin plus fluorouracil versus cisplatin plus paclitaxel in advanced head and neck cancer (E1395): An intergroup trial of the Eastern Cooperative Oncology Group. J Clin Oncol 23: 3562-3567

12. Vermorken JB, Mesia R, Rivera F, Remenar E, Kawecki A, et al. (2008) Platinum-based chemotherapy plus cetuximab in head and neck cancer. N Engl J Med 359: 1116-1127.

13. Haraf DJ, Weichselbaum RR, Vokes EE (1996) Re-irradiation with concomitant chemotherapy of unresectable recurrent head and neck cancer: a potentially curable disease. Ann Oncol 7: 913-918.

14. Kasperts N, Slotman BJ, Leemans CR, de Bree R, Doornaert P, et al. (2006) Results of postoperative reirradiation for recurrent or second primary head and neck carcinoma. Cancer 106: 1536-1547.

15. De Crevoisier R, Domenge C, Wibault P, Koscielny S, Lusinchi A, et al. (2001) Full dose reirradiation combined with chemotherapy after salvage surgery in head and neck carcinoma. Cancer 91: 2071-2076.

16. Milano MT, Vokes EE, Salama JK, Stenson KM, Kao J, et al. (2005) Twicedaily reirradiation for recurrent and second primary head-and-neck 
Citation: Martín EJ, Suarezb V, Cabrera IH, Peña LDL, Lentatia GO, et al. (2016) Reirradiation in Head and Neck Cancer: A Curative Intent in Recurrence or Second Tumors. J Nucl Med Radiat Ther 7: 281. doi:10.4172/2155-9619.1000281

Page 7 of 7

cancer with gemcitabine, paclitaxel, and 5-fluorouracil chemotherapy. Int J Radiat Oncol Biol Phys 61: 1096-1106.

17. Goldstein DP, Karnell LH, Yao M, Chamberlin GP, Nguyen TX, et al. (2008) Outcomes following reirradiation of patients with head and neck cancer. Head Neck 30: 765-770.

18. Langendijk JA, Kasperts N, Leemans CR, Doornaert P, Slotman BJ (2006) A phase II study of primary reirradiation in squamous cell carcinoma of head and neck. Radiother Oncol 78: 306-312.

19. Chua DT, Sham JS, Kwong PW, Hung KN, Leung LH (2003) Linear accelerator-based stereotactic radiosurgery for limited, locally persistent, and recurrent nasopharyngeal carcinoma: efficacy and complications. Int. J. Radiation Oncology Biol. Phys 56: 177-183.

20. Ozyigit G, Cengiz M, Yazici G, Yildiz F, Gurkaynak M, et al. (2011) A retrospective comparison of robotic stereotactic body radiotherapy and three-dimensional conformal radiotherapy for the reirradiation of local recurrent nasopharyngeal carcinoma. Int J Radiat Oncol Biol Phys 81: 263-268.

21. Spencer SA, Harris J, Wheeler RH, Machtay M, Schultz C, et al. (2008) Final report of RTOG 9610, a multi-institutional trial of reirradiation and chemotherapy for unresectable recurrent squamous cell carcinoma of the head and neck. Head Neck 30: 281-288.

22. Janot F, de Raucourt D, Benhamou E, Ferron C, Dolivet G, et al. (2008) Randomized trial of postoperative reirradiation combined with chemotherapy after salvage surgery compared with salvage surgery alone in head and neck carcinoma. J Clin Oncol 26: 5518-5523.
23. Langer CJ, Harris J, Horwitz EM, Nicolaou N, Kies M, et al. (2007) Phase II study of low-dose paclitaxel and cisplatin in combination with splitcourse concomitant twice-daily reirradiation in recurrent squamous cell carcinoma of the head and neck: results of Radiation Therapy Oncology Group Protocol 9911. J Clin Oncol 25: 4800-4805.

24. Kowalski LP (2002) Results of salvage treatment of the neck in patients with oral cancer. Arch Otolaryngol Head Neck Surg 128: 58-62.

25. Stevens KR Jr, Britsch A, Moss WT (1994) High-dose reirradiation of head and neck cancer with curative intent. Int J Radiat Oncol Biol Phys 29: 687-698.

26. Salama JK, Vokes EE, Chmura SJ, Milano MT, Kao J, et al. (2006) Longterm outcome of concurrent chemotherapy and reirradiation for recurrent and second primary head-and-neck squamous cell carcinoma. Int J Radiat Oncol Biol Phys 64: 382-391.

27. Choe KS, Haraf DJ, Solanki A, Cohen EE, Seiwert TY, et al. (2011) Prior chemoradiotherapy adversely impacts outcomes of recurrent and second primary head and neck cancer treated with concurrent chemotherapy and reirradiation. Cancer. Oct 117:4671-4678.

28. Ang KK, Jiang GL, Feng Y, Stephens LC, Tucker SL, et al. (2001) Extent and kinetics of recovery of occult spinal cord injury. Int J Radiat Oncol Biol Phys 50: 1013-1020.

29. Dawson LA, Myers LL, Bradford CR, Chepeha DB, Hogikyan ND, et al. (2001) Conformal re-irradiation of recurrent and new primary head-andneck cancer. Int J Radiat Oncol Biol Phys 50: 377-385. 